\title{
Quarks as Black holes
}

\author{
Muayyad A. Alobayde \\ Department of Physics \\ College of Science \\ University of Mosul
}

\author{
Issra A. Mohammed Ali \\ Department of Medical Instrument \\ Engineering \\ Technical College \\ Mosul
}

(Received 25/6/2012 ; Accepted 28/1/2013)

\begin{abstract}
The concept of the mass in (QCD) was discussed. The Schwarzschild radii and the Compton wave length of different types of quarks have been found. The comparison between the Schwarzschild radii with the proton and neutron radii shows that the quarks behave like a point particle inside the nucleon. It is found that the Compton wave lengths of the up and down quarks are of the order of the nucleon radii.
\end{abstract}

Keywords: Quark, the Schwarzschild radius, black holes.

\section{الكواركلت بومفها قوميلسوداء}

\section{المالخص}

تمت منالثة مفهوم الكتلة في الكرومودلينمك الكمي. و م اليجاد نصف قطرشوارزجايلد وطول موجة

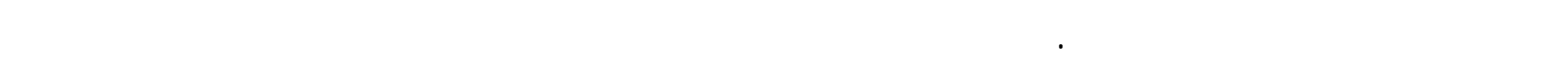

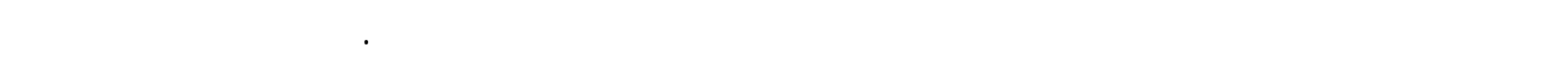
كومبتون للكواركلت من نوع اعلى ولسفل بحدود أنصف أقطار البروتون والنيترون. الكاملت الدالة: كوارك، نصف قطرشوارزجايلد، القوب للسوداء.

INTRODUCTION

From experiments, physicists learned that protons, neutrons, and other strongly interacting particles are made from quarks, antiquarks, and gluons. But we never see an isolated quark because; one never has enough energy to separate the quark and antiquark a macroscopic distance. The theoretical framework for analyzing quark confinement is the $S U$ (3) gauge theory of the strong interactions, known as Quantum Chromo dynamics QCD (Witten, 2001). 
On the other side, classically, a black hole absorbs everything that comes too close and does not emit anything, so we never see it. The theoretical framework for analyzing the black hole is the theory of General Relativity which describes the gravity.

Quantum mechanically, no black hole can exist, because if the Hamiltonian operator $H$ has a nonzero matrix element $<f|H| i>$ for absorption, then, as $H$ is hermitian, there is also a nonzero matrix element $<i|H| f>$ for emission.

In spite of the difference between quarks and black holes, many authors (Kov'acs et al., 2009) study the relation between them according to that; they are both part of nature, and so a theory of quantum gravity is needed to be described, but this is not simple because the mathematics of General Relativity is nonlinear and so does not satisfy the requirements of quantum theory.

But (Hawking, 1975) showed that quantum mechanically a black hole does emit thermal radiation. And, in cosmology, (Thakur, 2008) shows that a black hole cannot collapse to a singularity, instead it may end up as a quark star. (Kov'acs et al., 2009) investigate the possibility that stellar mass black holes, with masses in the range of $3.8 \mathrm{M}_{\text {sun }}$ and $6 \mathrm{M}_{\text {sun, }}$, respectively, could be in fact quark stars. (Siopsis, 2009) discuss the possibility that the quarkgluon plasma at strong coupling admits a description in terms of a black hole in asymptotically anti-de Sitter space.

In this work, we try to answer the question, is the quark a black hole, by using the condition for the black hole in the Schwarzschild solution to Einstein's field equations in the theory of general relativity, as the massive body which satisfy the relation (Narlikar, 1979):

$$
\frac{2 G M}{R c^{2}}=1
$$

Where, $\mathrm{G}$ is the gravitational constant, $\mathrm{M}$ is the mass of the body, $\mathrm{R}$ is the Schwarzschild radius, and $\mathrm{c}$ is the speed of the light at vacuum. Because of the difficulty which arises from the ambiguity in the notion of the mass of the quark in (QCD), we firstly discuss the quark mass before answering our question, then substituting the quarks masses for all the known quarks in equation (1) to find the Schwarzschild radius that make every quark a black hole. And calculating the Compton wave length for every quark to compare them with the radius of the nucleon, to see if they are compatible with them.

\section{Quark mass}

Unlike other fundamental interactions in nature such as electromagnetism, the strong interaction described by (QCD) is difficult to measure directly due to a property of this interaction known as quark confinement. The inability to measure the masses of these particles in an isolated system has made direct experimental inquiries of QCD difficult (Angerami and Cole, 2006).

Although one speaks of quark mass in the same way as the mass of any other particle, the notion of mass for quarks is complicated by the fact that quarks cannot be found free in nature, and are confined inside hadrons. So, the notion of quark masses is a theoretical construct which must be determined indirectly through their influence on hadronic properties (Göckeler et al., 2006). 
So, quantum field theory must be used to discuss quark masses at a fundamental level. The particle aspect of the quark is point-like even at scales thousands of times smaller than the proton size. The wave aspect of the quark extends over the size of the atomic nucleus. Light quark masses have not yet been well determined. This appears to be a consequence of quark confinement as well as the fact that the light quark masses are significantly lighter than the typical hadronic scale and as such their impact on most of the hadron masses or other properties is very small (Guo et al., 2010).

Heavy quark masses, charm, bottom and top quarks, are obtained from the masses of hadrons containing a single heavy quark and one or two light antiquarks light quarks and from the analysis of quarkonia. Lattice QCD computations using the heavy quark effective theory (HQET) or non-relativistic quantum chromo dynamics (NRQCD) are currently used to determine these quark masses. The top quark is sufficiently heavy that perturbative QCD can be used to determine its mass.

\section{Schwarz child's radius of the quark}

The Schwarzschild's solution of Einstein's gravitational field equations for spherical symmetric mass tell us that, a massive object is a black hole if it satisfies equation (1), which can be written as

$\mathrm{R}=1.48 \times 10^{-27} \mathrm{~m}$

$\mathrm{M}$ is the mass in $\mathrm{kg}$

or as

$R_{\mathrm{q}}=2.6344 \times 10^{-57} \mathrm{~m}$

$\mathrm{M}$ is the mass in $\mathrm{MeV}$ unit.

So, knowing the mass of the quark gives us the possibility to find the Schwarzschild radius that makes the quark a black hole. But as it was discussed previously the quark mass is not well defined, so, we take several estimated masses for each quark, Tables $(1,2)$,

Table 1: Light quark mass values (in unit of MeV) (Koide, 1994)

\begin{tabular}{|l|l|l|l|}
\hline Source & $\mathbf{m}_{\mathbf{u}}$ & $\mathbf{m}_{\mathbf{d}}$ & $\mathbf{m}_{\mathbf{s}}$ \\
\hline Okubo (1969) & 300 & 300 & 500 \\
\hline Veltman (1980) & 250 & 250 & 300 \\
\hline Grasser and Leutwyler (1982) & $5.1 \pm 1.5$ & $8.9 \pm 2.6$ & $175 \pm 55$ \\
\hline Domingues and Rafael (1987) & $5.6 \pm 1.1$ & $9.9 \pm 1.1$ & $199 \pm 33$ \\
\hline Narison (1989) & $5.2 \pm 0.5$ & $9.2 \pm 0.5$ & $159.5 \pm 8.8$ \\
\hline El Naschie (2002) & 5.236 & 8.47 & 179.4 \\
\hline Eidelman et al. (2004) & $1.5-4.0$ & $4-8$ & $80-130$ \\
\hline Allison (2005) & $1-5$ & $5-9$ & $80-130$ \\
\hline
\end{tabular}


Table 2: Heavy quark mass values (in unit of MeV) (Koide, 1994)

\begin{tabular}{|l|l|l|l|}
\hline Source & $\mathbf{m}_{\mathbf{c}}$ & $\mathbf{m}_{\mathbf{b}}$ & $\mathbf{m}_{\mathbf{t}}$ \\
\hline Okubo (1969) & 1700 & 5000 & 18000 \\
\hline Veltman (1980) & 1500 & 5000 & 20000 \\
\hline Gasser and Leutwyler (1982) & $1270 . \pm 0.05$ & $4250 \pm 0.10$ & \\
\hline El Naschie (2002) & 1270.820393 & 4236.067977 & 42360.67977 \\
\hline Eidelman et al. (2004) & $1150-1350$ & $4100-4400$ & 174300 \\
\hline Allison (2005) & $1150-1350$ & $4100-4400$ & 175000 \\
\hline
\end{tabular}

And we find Schwarz child's radii Tables $(3,4)$ that make the corresponding quarks a black hole.

\section{Table 3: Radius for light quarks (in unit of meters)}

\begin{tabular}{|l|l|l|l|}
\hline Source & $\mathbf{R}_{\mathbf{u}}$ & $\mathbf{R}_{\mathbf{d}}$ & $\mathbf{R}_{\mathbf{s}}$ \\
\hline Okubo (1969) & $7.9476 \times 10^{-55}$ & $7.947 \times 10^{-55}$ & $1.492 \times 10^{-54}$ \\
\hline Veltman (1980) & $6.6156 \times 10^{-55}$ & $6.6156 \times 10^{-55}$ & $7.9976 \times 10^{-55}$ \\
\hline $\begin{array}{l}\text { Grasser and Leutwyler } \\
(1982)\end{array}$ & $1.3510 \times 10^{-57}$ & $2.357 \times 10^{-56}$ & $4.635 \times 10^{-55}$ \\
\hline $\begin{array}{l}\text { Domingues and Rafael } \\
(1987)\end{array}$ & $1.4835 \times 10^{-56}$ & $2.622 \times 10^{-56}$ & $5.2717 \times 10^{-55}$ \\
\hline Narison (1989) & $1.3775 \times 10^{-56}$ & $2.436 \times 10^{-56}$ & $4.225 \times 10^{-55}$ \\
\hline El Naschie (2002) & $1.387 \times 10^{-56}$ & $2.2436 \times 10^{-56}$ & $4.7536 \times 10^{-55}$ \\
\hline Eidelman et al. (2004) & $\begin{array}{l}3.9738 \times 10^{-57} \\
1.05968 \times 10^{-56}\end{array}$ & $\begin{array}{l}1.0596 \times 10^{-56}- \\
2.1193 \times 10^{-56}\end{array}$ & $2.11936 \times 10^{-55}-3.4336 \times 10^{-55}$ \\
\hline Allison (2005) & $\begin{array}{l}2.6493 \times 10^{-57}- \\
1.3246 \times 10^{-56}\end{array}$ & $\begin{array}{l}1.324 \times 10^{-56}- \\
2.3842 \times 10^{-56}\end{array}$ & $2.11936 \times 10^{-55}-3.433 \times 10^{-55}$ \\
\hline
\end{tabular}

Table 4: Radius for heavy quarks (in unit of meters)

\begin{tabular}{|l|l|l|l|}
\hline Source & $\mathbf{R}_{\mathbf{c}}$ & $\mathbf{R}_{\mathbf{b}}$ & $\mathbf{R}_{\mathbf{t}}$ \\
\hline Okubo (1969) & $4.5036 \times 10^{-54}$ & $1.3246 \times 10^{-53}$ & $4.7685 \times 10^{-52}$ \\
\hline Veltman (1980) & $3.9738 \times 10^{-54}$ & $1.3246 \times 10^{-53}$ & $5.2984 \times 10^{-53}$ \\
\hline $\begin{array}{l}\text { Gasser and Leutwyler } \\
\text { (1982) }\end{array}$ & $3.3644 \times 10^{-54}$ & $1.1258 \times 10^{-53}$ & \\
\hline E1 Naschie (2002) & $3.3665 \times 10^{-54}$ & $1.122 \times 10^{-53}$ & $1.222 \times 10^{-52}$ \\
\hline Eidelman et al. (2004) & $3.046 \times 10^{-54}$ & $1.08617 \times 10^{-53}-1.1656 \times 10^{-53}$ & $4.617 \times 10^{-52}$ \\
\hline Allison (2005) & $3.576 \times 10^{-54}$ & & \\
\hline & $3.046 \times 10^{-54}-$ & $1.08617 \times 10^{-53}-1.1656 \times 10^{-53}$ & $4.636 \times 10^{-52}$ \\
\hline
\end{tabular}




\section{Compton wave length of the quark}

As a consequence of the duality behavior, any massive particles must have a Compton wave lengths associated with their masses which can be calculated using the known formula:

$$
\lambda=\frac{h}{M c}
$$

Or, by using $\left(\mathrm{h}=6.625 \times 10^{-34} \mathrm{~J} . \mathrm{s}, \mathrm{c}=3 \times 10^{8} \mathrm{~ms}^{-1}, \mathrm{MeV}=1.6 \times 10^{-13} \mathrm{~J}\right.$, and $\left.\mathrm{E}=\mathrm{Mc}^{2}\right)$ we can write

$$
\lambda=2.20980 \times 10^{-42} \mathrm{M}^{-1}
$$

With (M in $\mathrm{kg})$

Accordingly we can find the wave lengths associated with every quark, Tables $(5,6)$. As the nucleons compose of three quarks $\left(1 q_{d}+2 q_{u}\right)$ or $\left(1 q_{u}+2 q_{d}\right)$ one can compare the wave lengths of these quarks with the radius of the nucleons.

Table 5: wave length $\lambda$ for light quarks (in unit of meters)

\begin{tabular}{|l|l|l|l|}
\hline Source & $\lambda_{\mathrm{u}}$ & $\lambda_{\mathrm{d}}$ & $\lambda_{\mathrm{s}}$ \\
\hline Okubo (1969) & $4.1150 \times 10^{-15}$ & $4.1150 \times 10^{-15}$ & $2.469 \times 10^{-15}$ \\
\hline Veltman (1980) & $4.943 \times 10^{-15}$ & $4.943 \times 10^{-15}$ & $4.1150 \times 10^{-15}$ \\
\hline Grasser and Leutwyler (1982) & $2.4206 \times 10^{-13}$ & $1.387 \times 10^{-13}$ & $7.055 \times 10^{-15}$ \\
\hline Domingues and Rafael (1987) & $2.2045 \times 10^{-13}$ & $1.2470 \times 10^{-13}$ & $6.2038 \times 10^{-15}$ \\
\hline Narison (1989) & $2.3740 \times 10^{-13}$ & $1.3425 \times 10^{-13}$ & $7.740 \times 10^{-15}$ \\
\hline El Naschie (2002) & $2.3578 \times 10^{-13}$ & $1.457 \times 10^{-13}$ & $6.8800 \times 10^{-15}$ \\
\hline Eidelman et al., (2004) & $8.230 \times 10^{-13}$ & $3.086 \times 10^{-13}$ & $1.5453 \times 10^{-14}$ \\
& $3.086 \times 10^{-13}-$ & $1.54315 \times 10^{-13}$ & $9.525 \times 10^{-15}-$ \\
\hline Allison (2005) & $1.234 \times 10^{-12}$ & $2.4690 \times 10^{-15}$ & $1.5431 \times 10^{-14}$ \\
& $2.469 \times 10^{-13}$ & $1.3716 \times 10^{-15}$ & $9.525 \times 10^{-15}-$ \\
\hline
\end{tabular}

Table 6: wave length $\lambda$ for heavy quarks (in unit of meters)

\begin{tabular}{|l|l|l|l|}
\hline Source & $\lambda_{\mathbf{c}}$ & $\lambda_{\mathbf{b}}$ & $\lambda_{\mathrm{t}}$ \\
\hline Okubo (1969) & $7.2619 \times 10^{-16}$ & $2.469 \times 10^{-16}$ & $6.8584 \times 10^{-18}$ \\
\hline Veltman (1980) & $8.23016 \times 10^{-16}$ & $2.4690 \times 10^{-16}$ & $6.172 \times 10^{-17}$ \\
\hline Gasser and Leutwyler (1982) & $9.7206 \times 10^{-16}$ & $2.90495 \times 10^{-16}$ & \\
\hline El Naschie (2002) & $9.7176 \times 10^{-16}$ & $2.9143 \times 10^{-16}$ & $2.9143 \times 10^{-17}$ \\
\hline Eidelman et al. (2004) & $1.07350 \times 10^{-15}-$ & $3.01103 \times 10^{-16}$ & $7.0829 \times 10^{-18}$ \\
& $9.144 \times 10^{-16}-$ & $2.8057 \times 10^{-16}-$ & \\
\hline Allison (2005) & $1.07350 \times 10^{-15}-$ & $3.01103 \times 10^{-16}-$ & $7.0544 \times 10^{-18}$ \\
& $9.144 \times 10^{-16}-$ & $2.8057 \times 10^{-16}$ & \\
\hline
\end{tabular}




\section{RESULTS AND DISCUSSION}

It is clear from Tables $(1,2)$ that rest mass of the quarks is not well defined and that is because we can not find free quark. Tables $(3,4)$ show that the Schwarzschild radii of the quarks make them point-like particles and this agree with the particle aspect of the quark (Guo et al., 2010). The boundary conditions that governed the existence of the quarks inside the nucleon, that they are free particles surrounded by a very strong potential barrier, make them like particle in a box, the known quantum states, and so they behave as standing waves.

This expected behavior means that they can be in different energy states and that is why we take different masses to them. And it is shown from the first two rows of Table (5) that the nucleon radius $\left(R_{n}=5.751 \pm 0.175(\exp ) \pm 0.026 \mathrm{fm}, R_{n}-R_{p}=0.302 \pm 0.175 \mathrm{fm}\right)$ where $R_{p}$ is the point proton radius. (Horowitz et al., 2012) is comparable with the Compton wave length of the (up) and (down) quarks.

\section{REFERENCES}

Allison, I.; (2005). Dynamical lattice QCD determinations for heavy quark physics, Ph.D. Thesis, Dept of University of Glasgow, U.K.

Angerami, A.; Cole, B.A. (2006). Heavy Flavor Jet Structure in Relativistic Heavy Ion Collisions. Columbia Undergraduate Sci. J.,1, (1).

Dominguez, C.A.; deRafael, E. (1987). Light quark masses in QCD from local duality. Ann. Phys. 174, 372.

Eidelman, S.; Hayes, K.G.; Olive, K.A.; Aguilar-Benitez, M.; Amsler, C.; Asner, D.; Babu, K.S.; Barnett, R.M.; Beringer, J.; Burchat, P.R.; Carone, C.D.; Caso, C.; Conforto, G.; Dahl, O. ; D'Ambrosio, G.; Doser, M.; Feng, J.L.; Gherghetta, T.; Gibbons, L.; Goodman, M.; Groom, D.E. ; Gurtu, A. ; Hagiwara, K., Hernandez-Rey, J.J.; Hikasa, K.; Honscheid, K.; Jawahery, H., Kolda, C.; Kwon, Y.; Mangano, M.L.; Manohar, A.V.; March-Russell, J.; Masoni, A.; Miquel, R.; Monig, K. ; Murayama, H.; Nakamura, K.; Navas, S., Pape, L.; Patrignani, C.; Piepke, A., Raffelt, G.; Roos, M.; Tanabashi, M.; Terning, J.; Tornqvist, N.A.; Trippe, T.G.; Vogel, P.; Wohl, C.G.; Workman, R.L.; YaoW.-M.; Zyla, P.A. (2004). Particle data group. Phys. Lett. B 592(1).

El- Naschie, M.S. (2002). On the exact mass spectrum of quarks. Chaos, Solitons and Fractals, 14, 369-376.

Göckeler, M.; Horsley, R.; Irving, A.C.; Pleiter, D.; Rakow, P.E.L.; Schierholz, G.; Stüben, H.; Zanotti, J.M. (2006). Determing the strange quark mass for 2- flavor QCD, Nuclear Phys. B153, 154-161.

Gasser, J.; Leutwyler, H. (1982). Quark masses. Phys. Rep. 87, 77-197

Guo, F.; Hanhart, C.; Meißner, U. (2010). Extracting the light quark mass ratio mu/md from bottomonia transitions. arXiv:1007. 4682v2.

Hawking, S.W. (1975). Particle Creation by Black Holes. Common. Math. Phys. 43, 199-220.

Horowitz, C.J.; Ahmed, Z.; Jen, C.M.; Rakhman, A.; Souder, P.A. ; Dalton, M.M.; Liyanage, N.; Paschke, K.D.; Saenboonruang, K.; Silwal, R.; Franklin, G.B.; Friend, M.; Quinn, 
B.; Kumar, K.S.; McNulty, D.; Mercado, L.; Riordan, S.; Wexler, J.; Michaels, R.W.; Urciuoli, G.M. (2012). Weak charge form factor and radius of ${ }^{208} \mathrm{~Pb}$ through parity violation in electron scattering. Phys. Rev. C 85, 032501(R).

Koide, Y. (1994). Table of running Quark Mass Values: 1994, LaTex, 13, US-94-05.

Kov'acs, Z.; Cheng, K.S.; Harko, T. (2009). Can stellar mass black holes be quark stars?. MNRS, 400, 1632-1642.

Narlikar, J.V. (1979). "General Relativity And Cosmology”. The Macmillan Press LTD, London, $157 \mathrm{p}$.

Narison, S. (1989). Light and heavy quark masses. Phys. Lett. B216, 191.

Okubo, S. (1969). Asymptotic SU (6) W rules II: applications and bare Quark masses. Phys. Rev., 188, 2300-2307.

Siopsis, G. (2009). Black holes and the quark-gluon plasma. J. Phys: Conference Series. 189.

Thakur, R.K. (2008). "Do Black Holes End up as Quark Stars ?." Astronomy and Astrophysics manuscript no 2007AA 1 .

Veltman, M. (1980). Radioactive correction to vector boson masses. Phys. Lett. B 91(1).

Witten, E. (2001). Black holes and quark confinement. Current Sci., 81(12), 1576-1581. 